Triangulated categories and the Ziegler spectrum

Garkusha, Grigory and Prest, Mike

2005

MIMS EPrint: 2006.122

Manchester Institute for Mathematical Sciences

School of Mathematics

The University of Manchester

\footnotetext{
Reports available from: http://eprints.maths.manchester.ac.uk/

And by contacting: The MIMS Secretary

School of Mathematics

The University of Manchester

Manchester, M13 9PL, UK
} 


\title{
TRIANGULATED CATEGORIES AND THE ZIEGLER SPECTRUM
}

\author{
GRIGORY GARKUSHA AND MIKE PREST
}

\section{INTRODUCTION}

The Ziegler spectrum of a ring is a topological space defined in terms of its category of modules. The points are certain indecomposable modules and the topology, originally defined in model-theoretic terms [Zie], can be defined in a number of alternative but equivalent ways, see $[\mathrm{He} 2, \mathrm{Kr} 1]$ for example. In particular it may be defined as a topology on the set of indecomposable injective objects in an associated functor category. In [Kr4] Krause used this approach in order to define the Ziegler spectrum for any compactly generated triangulated category.

To any ring $R$ there is associated the triangulated category, $D(R)$, of unbounded complexes of $R$-modules. One may ask what is the relation between the Ziegler spectrum of $R$ and that of $D(R)$. We are able to give a complete answer when $R$ is right hereditary 8.1 or von Neumann regular 8.5. For general rings we have that a pure-injective complex has all cohomology modules pure-injective 7.1 and that the Ziegler spectrum of $D(R)$ contains countably many disjoint copies of the Ziegler spectrum of $R, 7.3$ but that in general there are further points in the spectrum of $D(R)$. As for modules there is a duality which induces an isomorphism 7.5 between the topologies on the spectra of $D(R)$ and $D\left(R^{\mathrm{op}}\right)$.

We also note 6.1 the relation between the Ziegler spectrum of a quasi-Frobenius ring and that of its stable module category.

We define a notion of absolutely pure relative to a fixed generator in any monogenic compactly generated triangulated category. For the case of $D(R)$ with generator $R$, we show that all cohomology modules of an absolutely pure complex are absolutely pure and, in case $R$ is coherent, we show that a complex is absolutely pure iff it is homologically isomorphic to a coproduct of absolutely pure modules (regarded as complexes concentrated in a single degree) 5.2 .

We also show 4.4 that the topology of the Ziegler spectrum of a compactly generated triangulated category may be defined in the style of [Zie], that is, using positive primitive formulas. For that we first set up the canonical language of such a category. We show that the category has elimination of quantifiers in this language 3.2.

2000 Mathematics Subject Classification. Primary 18E30; Secondary 03C60.

This paper was written during the visit of the first author to the University of Manchester supported by EPSRC grant GR/R94114/01. He would like to thank the University for the kind hospitality. 


\section{Compactly generated triangulated CAtegories}

We fix a triangulated category $\mathcal{T}$ with arbitrary direct sums. An object $X$ of $\mathcal{T}$ is said to be compact if for every family $\left\{Y_{i}\right\}_{i \in I}$ of objects from $\mathcal{T}$ the canonical map

$$
\bigoplus_{i \in I} \mathcal{T}\left(X, Y_{i}\right) \longrightarrow \mathcal{T}\left(X, \bigoplus_{i \in I} Y_{i}\right)
$$

is an isomorphism. The suspension of any compact object is compact. The category $\mathcal{T}$ is compactly generated if there exists a set $\mathcal{C}$ of compact objects of $\mathcal{T}$ such that $\mathcal{T}(\mathcal{C}, Y)=0$ (i.e. $\mathcal{T}(C, Y)=0$ for all $C \in \mathcal{C}$ ) implies $Y=0$ for every object $Y$ in $\mathcal{T}$. We refer to such a set $\mathcal{C}$ as a generating set if it is closed under suspension, for which we write $\mathcal{C}=\Sigma \mathcal{C}$. The triangulated subcategory of $\mathcal{T}$ consisting of compact objects will be denoted by $\mathcal{T}^{c}$. We observe that $\mathcal{T}^{c}$ is the smallest triangulated subcategory in $\mathcal{T}$ containing any generating set. Also $\mathcal{T}$ is closed under taking direct products.

The following examples of such categories are particularly important for applications:

(1) the derived category $D(R)$ of unbounded complexes of modules for a ring $R$;

(2) the stable module category $\underline{\operatorname{Mod}} \Lambda$ of a QF-ring $\Lambda$;

(3) the stable homotopy category $\mathrm{Ho}(\mathcal{S})$ of CW-spectra.

One can specify in each case generating sets and the compact objects.

(1) The set $\mathcal{R}=\{R[n]\}_{n \in \mathbf{Z}}$ generates $D(R)$, where $R[n]$ denotes the complex concentrated in the $-n$th degree, and the perfect complexes (i.e. the complexes isomorphic to bounded complexes of finitely generated projective modules) are the compact objects in $D(R)$;

(2) If $\left\{S_{1}, \ldots, S_{l}\right\}$ is the set of the simple $\Lambda$-modules, then the set

$$
\mathcal{R}=\left\{\Omega^{n} S_{1}\right\}_{n \in \mathbf{Z}} \bigcup \ldots \bigcup\left\{\Omega^{n} S_{l}\right\}_{n \in \mathbf{Z}}
$$

of suspensins of the simple modules generates $\underline{\operatorname{Mod}} \Lambda$, and the finitely generated modules are the compact objects in $\underline{\operatorname{Mod}} \Lambda$;

(3) The set $\mathcal{R}=\left\{S^{n}\right\}_{n \in \mathbf{Z}}$ of suspensions of the sphere spectrum $S^{0}$ generates $\mathrm{Ho}(\mathcal{S})$, and the finite spectra are the compact objects in $\mathrm{Ho}(\mathcal{S})$.

Following Hovey, Palmieri and Strickland [HPS], we call a triangulated category $\mathcal{T}$ monogenic if there is an object $X$ in $\mathcal{T}$ such that the only localizing subcategory in $\mathcal{T}$ containing $X$ is $\mathcal{T}$ itself, where a localising subcategory of $\mathcal{T}$ is a triangulated subcategory which is closed under arbitrary coproducts (and hence under direct summands). We shall refer to $X$ as a generator. All of the above triangulated categories are plainly monogenic.

If $\mathcal{T}$ is a compactly generated triangulated category then, following Krause [Kr3], a triangle $L \stackrel{\alpha}{\longrightarrow} M \stackrel{\beta}{\longrightarrow} N \longrightarrow \Sigma L$ in $\mathcal{T}$ is pure-exact if for every compact $C$ in $\mathcal{T}^{c}$ the induced sequence $0 \longrightarrow \mathcal{T}(C, L) \longrightarrow \mathcal{T}(C, M) \longrightarrow \mathcal{T}(C, N) \longrightarrow 0$ is exact. We call the map $\alpha$ a pure monomorphism. The object $L$ of $\mathcal{T}$ is pure-injective if every pure-exact triangle $L \longrightarrow M \longrightarrow N \longrightarrow \Sigma L$ is split.

Consider, for a compactly generated triangulated category $\mathcal{T}$, the category Mod $\mathcal{T}^{c}$ of additive contravariant functors from $\mathcal{T}^{c}$ to Ab. We have a natural functor $H: \mathcal{T} \longrightarrow \operatorname{Mod} \mathcal{T}^{c}$ sending $X$ to $H_{X}=\left.\mathcal{T}(-, X)\right|_{\mathcal{T}^{c}}$. A $\mathcal{T}^{c}$-module $M$ (that is, an object of Mod $\mathcal{T}^{c}$ ) is finitely generated if there exists an exact sequence $H_{X} \longrightarrow M \longrightarrow 0$ for some $X$ in $\mathcal{T}^{c}$, and $M$ is finitely presented if there exists an exact sequence $H_{Y} \longrightarrow H_{X} \longrightarrow M \longrightarrow 0$ with $X$ and 
$Y$ in $\mathcal{T}^{c}$. We denote the full subcategory of finitely presented objects by $\bmod \mathcal{T}^{c}$. Below we list some basic facts about the category $\operatorname{Mod} \mathcal{T}^{c}$.

$\diamond \operatorname{Mod} \mathcal{T}^{c}$ is a locally coherent Grothendieck category, because any map $Y \longrightarrow Z$ in $\mathcal{T}^{c}$ has a weak kernel $X \longrightarrow Y$, i.e. the sequence $H_{X} \longrightarrow H_{Y} \longrightarrow H_{Z}$ is exact. Equivalently, the category $\bmod \mathcal{T}^{c}$ is abelian.

$\diamond$ A triangle $L \longrightarrow M \longrightarrow N \longrightarrow \Sigma L$ is pure-exact iff the sequence $0 \longrightarrow H_{L} \longrightarrow$ $H_{M} \longrightarrow H_{N} \longrightarrow 0$ is exact.

$\diamond$ The functor $H$ restricts to an identification of the pure-injective objects of $\mathcal{T}$ with the injective objects of $\operatorname{Mod} \mathcal{T}^{c}$ [Kr3]. The (iso classes of) indecomposable injective objects of $\operatorname{Mod} \mathcal{T}^{c}$ form a set since every indecomposable injective $\mathcal{T}^{c}$-module arises as an injective hull of a finitely generated $\mathcal{T}^{c}$-module. Therefore, the (iso classes of) indecomposable pure-injective objects of $\mathcal{T}$ form a set which we denote by Zsp $\mathcal{T}$.

We say that an object $M$ of $\operatorname{Mod} \mathcal{T}^{c}$ is absolutely pure if $\operatorname{Ext}^{1}(F, M)=0$ for all $F \in \bmod \mathcal{T}^{c}$. For example, every functor $H_{X}$ with $X$ an object of $\mathcal{T}$ is absolutely pure $[\operatorname{Kr} 3$, 1.6]. Clearly, every absolutely pure object $M$ of $\operatorname{Mod} \mathcal{T}^{c}$ is a cohomological functor on $\mathcal{T}^{c}$.

The category of finitely presented $\mathcal{T}^{c}$-modules, mod $\mathcal{T}^{c}$, has enough injectives. Indeed, every module $H_{X}$ with $X$ compact is injective in $\bmod \mathcal{T}^{c}$ since it is absolutely pure.

Let $F$ be an arbitrary object of $\bmod \mathcal{T}^{c}$ and let

$$
H_{X} \stackrel{H_{\alpha}}{\longrightarrow} H_{Y} \longrightarrow F \longrightarrow 0
$$

be a projective presentation of $F$ with $X$ and $Y$ compact. Complete $\alpha$ to a triangle

$$
X \stackrel{\alpha}{\longrightarrow} Y \longrightarrow Z \longrightarrow \Sigma X \text {. }
$$

Then,

$$
0 \longrightarrow F \longrightarrow H_{Z} \longrightarrow H_{\Sigma X}
$$

is an injective presentation of $F$ in $\bmod \mathcal{T}^{c}$. Since $\mathcal{T}^{c}$ is closed under direct summands, the functor $H$ identifies compact objects with injectives in $\bmod \mathcal{T}^{c}$.

The pure-injective objects in a triangulated category have a characterization similar to that in [JeLe] of pure-injective modules.

Theorem 2.1 (Krause [Kr3]). An object $L$ in $\mathcal{T}$ is pure-injective iff for every set $I$ the summation map $L^{(I)} \longrightarrow L$ factors through the canonical map $L^{(I)} \longrightarrow L^{I}$ from the coproduct to the product.

Example. (1) Let $\underline{\operatorname{Mod}} \Lambda$ be the stable category of a QF-ring $\Lambda$. An object $L$ in $\underline{\operatorname{Mod}} \Lambda$ is pure-injective iff it is a pure-injective $\Lambda$-module [Kr3, 1.16].

(2) Every endofinite object in $\mathcal{T}$ (in the sense of $[\mathrm{Kr} 2, \mathrm{KR}]$ ) is pure-injective $[\mathrm{KR}]$. For example, every perfect complex $P$ in $D(\Lambda)$ over an artin algebra $\Lambda$ is endofinite, because the cohomology $H^{n}(P)$ is an endofinite module for each $n \in \mathbf{Z}$.

Below we shall show that any complex $L[n]$ concentrated in the $-n$th degree with $L$ a pure-injective $R$-module is a pure-injective object in the derived category $D(R)$.

We call a map $\varphi: X \longrightarrow \widehat{X}$ a pure-injective hull of $X$ if $\varphi$ is a pure monomorphism, $\widehat{X}$ is pure-injective and every endomorphism $\psi$ of $\widehat{X}$ satisfying $\psi \varphi=\varphi$ is an isomorphism. Every object $X$ in $\mathcal{T}$ admits a pure-injective hull $\varphi: X \longrightarrow \widehat{X}$ since $\varphi: X \longrightarrow \widehat{X}$ is a pure-injective hull iff $H_{\varphi}: H_{X} \longrightarrow H_{\widehat{X}}$ is an injective hull in Mod $\mathcal{T}^{c}$ [Kr3, 1.12] and since 
Mod $\mathcal{T}^{c}$ has injective hulls. If $\varphi^{\prime}: X \longrightarrow \widehat{X}^{\prime}$ is another injective hull, then there exists an isomorphism $\psi: \widehat{X} \longrightarrow \widehat{X}^{\prime}$ such that $\varphi^{\prime}=\psi \varphi[\mathrm{Kr} 3]$.

Lemma 2.2. A pure-injective object $X$ of $\mathcal{T}$ is indecomposable iff the endomorphism ring $\operatorname{End}_{\mathcal{T}} X$ of $X$ is local.

Proof. By $[\mathrm{Kr} 3,1.8] \operatorname{End}_{\mathcal{T}} X=\operatorname{End}_{\mathrm{Mod}} \mathcal{T}^{c} H_{X}$. Since $H_{X}$ is injective in Mod $\mathcal{T}^{c}$ the assertion follows from the fact that an injective object in a functor category is indecomposable iff its endomorphism ring is local.

Theorem 2.3 (as Herzog [He2]). A pure-injective hull $\widehat{M}$ of a compact object $M$ is indecomposable iff the endomorphism ring $\operatorname{End}_{\mathcal{T}} M$ of $M$ is local. If, furthermore, $N$ is another compact object such that $\widehat{N} \simeq \widehat{M}$ then $N \simeq M$.

Proof. We repeat Herzog's arguments for the convenience of the reader. The mod $\mathcal{T}^{c}$ injective object $H_{M}$ has a local endomorphism $\operatorname{ring} \operatorname{End}_{\bmod \mathcal{T}^{c}} H_{M}=\operatorname{End}_{\mathcal{T}} M$ and hence is indecomposable. Because the category $\bmod \mathcal{T}^{c}$ has enough injectives, $H_{M}$ is, therefore, a uniform $\bmod \mathcal{T}^{c}$-object. Hence it is uniform in $\operatorname{Mod} \mathcal{T}^{c}$ and so its injective hull $E\left(H_{M}\right)=H_{\widehat{M}}$ is indecomposable.

On the other hand, if $\widehat{M}$ is indecomposable then the injective $\mathcal{T}^{c}$-module $H_{\widehat{M}}$ is indecomposable. Hence $H_{M} \leq H_{\widehat{M}}$ is a uniform $\bmod \mathcal{T}^{c}$-injective. The proof of [St, V.5.1] then shows that $\operatorname{End}_{\mathcal{T}} M$ is a local ring.

If furthermore $N$ is another compact object such that $\widehat{N} \simeq \widehat{M}$, then both $H_{N}$ and $H_{M}$ are essential extensions of some finitely generated, hence finitely presented, subobject $F$. Then $H_{N} \simeq E_{\bmod \mathcal{T}^{c}}(F) \simeq H_{M}$. So $N \simeq M$, as claimed.

Below we shall need the following lemma.

Lemma 2.4. Let $X \longrightarrow Y \longrightarrow Z \longrightarrow X[1]$ be a pure-exact triangle of complexes in the derived category $D(R)$ of a ring $R$. Then the sequence of $R$-modules $0 \longrightarrow H^{n}(X) \longrightarrow$ $H^{n}(Y) \longrightarrow H^{n}(Z) \longrightarrow 0$ is pure-exact for all $n \in \mathbf{Z}$.

Proof. By assumption, the sequence

$$
\varepsilon: 0 \longrightarrow H_{X} \longrightarrow H_{Y} \longrightarrow H_{Z} \longrightarrow 0
$$

is exact in $\operatorname{Mod} \mathcal{P}$, where $\mathcal{P}$ is the category of perfect (=compact) complexes in $D(R)$. By $[\mathrm{Kr} 4,2.8] \varepsilon=\lim _{i} \varepsilon_{i}$ is a direct limit of split exact sequences

$$
\varepsilon_{i}: 0 \longrightarrow H_{X_{i}} \longrightarrow H_{Y_{i}} \longrightarrow H_{Z_{i}} \longrightarrow 0
$$

with $X_{i}, Y_{i}, Z_{i}$ some complexes in $D(R)$. Since $H^{n}(X)=H_{X}(R[-n])$ for any complex $X$, we see that the short exact sequence

$$
H^{n}(\varepsilon): 0 \longrightarrow H^{n}(X) \longrightarrow H^{n}(Y) \longrightarrow H^{n}(Z) \longrightarrow 0
$$

is the direct limit $H^{n}(\varepsilon)=\underline{\lim } H^{n}\left(\varepsilon_{i}\right)$ of split exact sequences

$$
H^{n}\left(\varepsilon_{i}\right): 0 \longrightarrow H^{n}\left(X_{i}\right) \longrightarrow H^{n}\left(Y_{i}\right) \longrightarrow H^{n}\left(Z_{i}\right) \longrightarrow 0,
$$

so is pure, as required. 
The Ziegler spectrum of the category $\mathcal{T}$ has for its points those of the set Zsp $\mathcal{T}$. The Ziegler topology for $\operatorname{Mod} R$ was originally defined in terms of pp formulas, equivalently in terms of the pp-definable subgroups (=subgroups of finite definition) that such formulas define. We will show that it may be defined in the same way for compactly generated triangulated categories. If we are to make sense of pp formulas in this context we must set up an appropriate language for $\mathcal{T}$ : this will be done below.

Let $\alpha: G \longrightarrow H$ be a morphism in $\mathcal{T}^{c}$. Then for any $M$ in $\mathcal{T}$ we have the induced map $\mathcal{T}(\alpha, M): \mathcal{T}(H, M) \longrightarrow \mathcal{T}(G, M)$ given by composition with $\alpha$. We write $M \alpha$ for the image of $\mathcal{T}(\alpha, M)$ and refer to this as a subgroup of finite definition of $M$ of sort $G$.

Define an order on such functors $(M \mapsto M \alpha)$ by setting $\beta \leq \alpha$, where $\alpha: G \longrightarrow H$ and $\beta: G \longrightarrow K$ are in $\mathcal{T}^{c}$, if for every $M$ in $\mathcal{T}$ we have $M \beta \leq M \alpha$. Taking $M=K$ we obtain the following.

Lemma 2.5. Given $\alpha: G \longrightarrow H$ and $\beta: G \longrightarrow K$ in $\mathcal{T}^{c}$, we have $\beta \leq \alpha$ iff there is $\nu: H \longrightarrow K$ with $\beta=\nu \alpha$.

We will show that these subgroups of finite definition coincide with the pp-definable subgroups in the natural language for $\mathcal{T}$.

\section{The language of a COMPACTly Generated tRiangulated CATEGORY}

Let $\mathcal{T}$ be a compactly generated triangulated category and let $\mathcal{T}^{c}$ be the subcategory of compact objects. In this section we define a corresponding (multi-sorted) language for $\mathcal{T}$.

For each object $G$ in $\mathcal{T}^{c}$ we introduce a sort and we say, for $X$ in $\mathcal{T}$, that the elements of $X$ of sort $G$ are the elements of $\mathcal{T}(G, X)$. We equip each sort with a symbol for addition and a symbol for 0 . Any given variable ranges over the elements of a single sort and we use a subscript to denote that sort: thus $v_{G}$, when interpreted in $X$, is a variable for elements of $\mathcal{T}(G, X)$.

For each morphism $\alpha: G \longrightarrow H$ in $\mathcal{T}^{c}$ we introduce a function symbol which, when interpreted, will be the map which we have seen just above, "composition with $\alpha$ ", from sort $H$ to sort $G$. We will use the same symbol for the function symbol as for the function (as usual).

Denote the resulting language by $L^{\mathcal{T}}$. We will call this language the canonical language of $\mathcal{T}$.

Let $\operatorname{Ax}(\mathcal{T})$ be a set of axioms expressing the positive atomic diagram (the "addition and multiplication tables") of $\mathcal{T}^{c}$, including the specification that all functions are additive. For example if $\alpha: G \longrightarrow H$ and $\beta: H \longrightarrow K$ are in $\mathcal{T}^{c}$ and if $\gamma=\beta \alpha$ then we put the axiom $\forall v_{K}\left(v_{K} \gamma=v_{K} \beta \alpha\right)$ into $\operatorname{Ax}(\mathcal{T})$. Then it is easy to see that the category of models for $\operatorname{Ax}(\mathcal{T})$ (where the morphisms are the $L^{\mathcal{T}}$-structure-preserving maps) is the category of right $\mathcal{T}^{c}$-modules - that is, the category, Mod $\mathcal{T}^{c}$, of functors. We regard the objects of $\mathcal{T}$ as structures for this language via the functor $H$ which takes $X$ to $\mathcal{T}(-, X)$ (recall that $H$ is faithful on objects, though not on morphisms).

Thus we have a multi-sorted first order language and so all the usual definitions and theorems of model theory apply. In particular we define pp formulas in the usual way (as 
formulas in the closure of the set of equations under conjunction and existential quantification) and so obtain a notion of "pp-definable subgroup" (the set of solutions of a pp formula) of an object of $\mathcal{T}$.

Note that what we termed the subgroups of finite definition are exactly those which are definable by a pp formula of the form " $\alpha \mid v_{G}$ " that is, of the form " $\exists v_{H}\left(v_{G}=v_{H} \alpha\right)$ " where $\alpha: G \longrightarrow H$ is in $\mathcal{T}^{c}$.

Proposition 3.1. Every pp formula in the language of $\mathcal{T}$ is equivalent to one of the form $\alpha \mid v$.

Proof. First we show that every quantifier-free formula $v_{G} \beta=0$ is equivalent to one of the given form (since $\mathcal{T}^{c}$ is closed under finite direct sums we may always suppose that a formula has at most a single free variable). We use the fact that $\mathcal{T}^{c}$ has pseudocokernels.

Suppose that $\beta: K \longrightarrow G$ is in $\mathcal{T}^{c}$. Complete $\beta$ to a triangle in $\mathcal{T}^{c}$

$$
K \stackrel{\beta}{\longrightarrow} G \stackrel{\alpha}{\longrightarrow} H \longrightarrow \Sigma K .
$$

Then $\alpha: G \longrightarrow H$ is a pseudocokernel of $\beta$ in $\mathcal{T}^{c}$. The formula $v_{G} \beta=0$ is equivalent to $\alpha \mid v_{G}$. For if $\alpha \mid v_{G}\left(\operatorname{read} v_{G}\right.$ now as a typical element of sort $\left.\mathcal{T}(G,-)\right)$, say $v_{G}=v_{H} \alpha$, then $v_{G} \beta=v_{H} \alpha \beta=0$ and, conversely, if $v_{G} \beta=0$ then, by definition of pseudocokernel, we have that $v_{G}$ factors through $\alpha$, as required.

Now take a general pp formula with free variable $v_{G}: \exists v_{G^{\prime}}\left(v_{G} \beta+v_{G^{\prime}} \beta^{\prime}=0\right)$. This is equivalent to the formula $\exists v_{G^{\prime}}\left(v_{G}, v_{G^{\prime}}\right)\left(\beta, \beta^{\prime}\right)^{T}=0$ where $\left(v_{G}, v_{G^{\prime}}\right)$ is regarded as a variable of type $G \oplus G^{\prime}$ and where $\left(\beta, \beta^{\prime}\right)^{T}$ is the obvious map to $G \oplus G^{\prime}$. By the above $\left(v_{G}, v_{G^{\prime}}\right)\left(\beta, \beta^{\prime}\right)^{T}=0$ is equivalent to a formula of the form $\alpha_{1} \mid\left(v_{G}, v_{G^{\prime}}\right)$ for some morphism $\alpha_{1}=\left(\alpha, \alpha^{\prime}\right): G \oplus G^{\prime} \longrightarrow H$ in $\mathcal{T}^{c}$. So our original formula is equivalent to $\exists v_{G^{\prime}} \exists v_{H}\left(v_{H} \alpha_{1}=\right.$ $\left.\left(v_{G}, v_{G^{\prime}}\right)\right)$ that is, to $\exists v_{H} \exists v_{G^{\prime}}\left(v_{H} \alpha=v_{G} \wedge v_{H} \alpha^{\prime}=v_{G^{\prime}}\right)$ which, in turn, is equivalent to $\exists v_{H}\left(v_{H} \alpha=v_{G}\right)$, as required.

As a consequence of the preceding proposition we see that pp-definable subgroups coincide with what we called subgroups of finite definition.

Since $\mathcal{T}^{c}$ has pseudokernels then, as in the first part of the proof above, every divisibility formula $\alpha \mid v$ is equivalent to an annihilation formula (one of the form $v \beta=0$ ). Thus one obtains elimination of quantifiers in the canonical language of $\mathcal{T}$.

Proposition 3.2. Let $\mathcal{T}$ be a compactly generated triangulated category. Then $\mathcal{T}$ has elimination of quantifiers in its canonical language.

We could now try to mimick the usual definition of the Ziegler topology on the set Zsp T of indecomposable pure-injective objects in $\mathcal{T}$ by taking as basic open sets those of the form $(\alpha / \beta)=\{N \in \mathrm{Zsp} \mathcal{T} \mid N \alpha>N \beta\}$ where $\alpha, \beta$ are morphisms in $\mathcal{T}^{c}$ with the same domain and with $\alpha>\beta$. It is not immediately clear, however, that we do get a basis for a topology: given $N \in(\alpha / \beta) \cap\left(\alpha^{\prime} / \beta^{\prime}\right)$ is there $\left(\alpha^{\prime \prime} / \beta^{\prime \prime}\right)$ with $N \in\left(\alpha^{\prime \prime} / \beta^{\prime \prime}\right) \subseteq(\alpha / \beta) \cap\left(\alpha^{\prime} / \beta^{\prime}\right)$ ?

We will resolve this indirectly as follows. One can define the Ziegler topology by using coherent functors on $\mathcal{T}$, as has been done in [Kr4], and one can show that the above basis property holds if we use coherent functors. Then we show that the topologies coincide. We shall show, moreover, that every basic open set $(\alpha / \beta)$ coincides with an open set of the form 
$(\delta)=\{N \in \operatorname{Zsp} \mathcal{T} \mid \delta(N) \neq 0\}$ where $\delta$ is a pp formula. Thus the Ziegler topology can be defined by using single pp formulas rather than pairs of formulas.

\section{Coherent Functors}

Let $\mathcal{T}$ be a compactly generated triangulated category. A functor $C: \mathcal{T} \longrightarrow \mathbf{A b}$ to the category of abelian groups is said to be coherent if there exists an exact sequence

$$
\mathcal{T}(A,-) \longrightarrow \mathcal{T}(B,-) \longrightarrow C \longrightarrow 0
$$

where $A$ and $B$ are compact objects. This notion extends that of coherent functor on the category of modules $\operatorname{Mod} R[\mathrm{Au}]$. The collection of all coherent functors $\mathcal{T} \longrightarrow \mathbf{A b}$ we denote by $\operatorname{coh} \mathcal{T}$. The category $\operatorname{coh} \mathcal{T}$ is abelian if we take as maps the natural transformations.

We say that a full subcategory $\mathcal{C}$ of $\mathcal{T}$ is definable if $\mathcal{C}=\left\{X \in \mathcal{T} \mid C_{i}(X)=0\right.$ for all $i \in$ $I\}$ for some family $\left(C_{i}\right)_{i \in I}$ of coherent functors. A subset $\mathcal{U}$ in Zsp $\mathcal{T}$ is Ziegler-closed if $\mathcal{U}=\mathcal{C} \cap Z \operatorname{sp} \mathcal{T}$ for some definable subcategory $\mathcal{C}$ of $\mathcal{T}$.

Theorem 4.1 (Krause [Kr4]). There are bijections between

$\diamond$ the set of definable subcategories $\mathcal{C}$ of $\mathcal{T}$,

$\diamond$ the set of Ziegler-closed subsets $\mathcal{U}$ of Zsp $\mathcal{T}$,

$\diamond$ the set of Serre subcategories $\mathcal{S}$ of $\operatorname{coh} \mathcal{T}$.

These bijections are defined as follows:

$$
\begin{aligned}
& \mathcal{C} \mapsto\left\{\begin{aligned}
\mathcal{U} & =\mathcal{C} \cap Z \operatorname{sp} \mathcal{T} \\
\mathcal{S} & =\{C \in \operatorname{coh} \mathcal{T} \mid C(X)=0 \text { for all } X \in \mathcal{C}\}
\end{aligned}\right. \\
& \mathcal{U} \mapsto\left\{\begin{aligned}
\mathcal{C} & =\left\{X \in \mathcal{T} \mid \text { there are } Y_{i} \in \mathcal{U} \text { and a pure triangle } X \rightarrow \prod_{i} Y_{i} \rightarrow Z \rightarrow \Sigma X\right\} \\
\mathcal{S} & =\{C \in \operatorname{coh} \mathcal{T} \mid C(X)=0 \text { for all } X \in \mathcal{U}\}
\end{aligned}\right. \\
& \mathcal{S} \mapsto\left\{\begin{aligned}
\mathcal{C} & =\{X \in \mathcal{T} \mid C(X)=0 \text { for all } C \in \mathcal{S}\} \\
\mathcal{U} & =\{X \in \mathrm{Zsp} \mathcal{T} \mid C(X)=0 \text { for all } C \in \mathcal{S}\}
\end{aligned}\right.
\end{aligned}
$$

The collection of Ziegler-closed subsets of Zsp $\mathcal{T}$ satisfies the axioms for the closed sets of a topology on Zsp $\mathcal{T}$. This topological space is called the Ziegler spectrum of $\mathcal{T}$.

Corollary 4.2. The collection of subsets of Zsp $\mathcal{T}$

$$
\mathcal{O}(C)=\{N \in \operatorname{Zsp} \mathcal{T} \mid C(N) \neq 0\}
$$

with $C \in \operatorname{coh} \mathcal{T}$ is a basis of open subsets of the Ziegler spectrum.

Proof. Clearly these sets are open. Every open subset in Zsp $\mathcal{T}$ is of the form

$$
\mathcal{O}(\mathcal{S})=\{N \in \mathrm{Zsp} \mathcal{T} \mid C(N) \neq 0 \text { for some } C \in \mathcal{S}\}
$$

with $\mathcal{S}$ a Serre subcategory in $\operatorname{coh} \mathcal{T}$. Then $\mathcal{O}(\mathcal{S})=\bigcup_{C \in \mathcal{S}} \mathcal{O}(C)$, as required.

The correspondence of Theorem 4.1 is proved by using the category of finitely presented objects, $\bmod \mathcal{T}^{c}$, of the category $\operatorname{Mod} \mathcal{T}^{c}$ and duality. Given a finitely presented object $F$ in $\operatorname{Mod} \mathcal{T}^{c}$ we define a coherent functor $D F: \mathcal{T} \longrightarrow \mathbf{A b}$ by

$$
D F(X)=\operatorname{Hom}\left(F, H_{X}\right) .
$$

The assignment $F \longmapsto D F$ puts the categories $\bmod \mathcal{T}^{c}$ and $\operatorname{coh} \mathcal{T}$ in duality $[\mathrm{Kr} 4,7.2]$. In particular, the functor $D$ sends Serre subcategories of $\bmod \mathcal{T}^{c}$ to those of $\operatorname{coh} \mathcal{T}$. 
Let SpMod $\mathcal{T}^{c}$ denote the set of (iso classes of) indecomposable injective objects in $\operatorname{Mod} \mathcal{T}^{c}$. As we have said already the functor $H: \mathcal{T} \longrightarrow \operatorname{Mod} \mathcal{T}^{c}$ restricts to an identification of the pure-injective objects in $\mathcal{T}$ with the injective objects in Mod $\mathcal{T}^{c}$. In particular, $H$ identifies the set Zsp $\mathcal{T}$ with the set, Sp Mod $\mathcal{T}^{c}$, of indecomposable injectives of Mod $\mathcal{T}^{c}$. The category Mod $\mathcal{T}^{c}$ is locally coherent. Therefore we can introduce the Ziegler topology on Zsp $\mathcal{T}$ by applying the definition of the Ziegler topology for locally coherent categories which is due to Herzog (see [He2, Kr1]). The closed subsets are defined as follows. Given a Serre subcategory $\mathcal{S}$ in $\bmod \mathcal{T}^{c}$ a subset

$$
\mathbf{U}(\mathcal{S})=\left\{X \in \operatorname{Zsp} \mathcal{T} \mid \operatorname{Hom}\left(\mathcal{S}, H_{X}\right)=0\right\}
$$

is, by definition, a closed subset in Zsp $\mathcal{T}$. The assignment $\mathcal{S} \longmapsto \mathbf{U}(\mathcal{S})$ induces a 1-1 correspondence between the set of Serre subcategories of $\bmod \mathcal{T}^{c}$ and the set of closed subsets of Zsp $\mathcal{T}$ (see [He2, Kr1]). Clearly, the set of closed subsets (1) coincides with the set of Ziegler-closed subsets defined above in terms of coherent functors.

The following lemma gives a relationship between pp formulas and coherent functors.

Lemma 4.3. Suppose that $\varphi$ is a pp formula. Then the assignment $M \longmapsto \varphi(M)$ defines a coherent functor $\mathcal{T} \longrightarrow \mathbf{A b}$, and any coherent functor arises in this way.

Proof. By Proposition 3.1 every pp formula in the language of $\mathcal{T}$ is equivalent to one of the form $\alpha \mid v_{G}$ with $\alpha: G \longrightarrow H$ a map in $\mathcal{T}^{c}$. Complete $\alpha$ to a triangle

$$
G \stackrel{\alpha}{\longrightarrow} H \longrightarrow K \longrightarrow \Sigma G \text {. }
$$

Then the functor $M \longrightarrow \varphi(M)$ is the coherent functor $M \longmapsto \operatorname{Coker}(\mathcal{T}(K, M) \rightarrow \mathcal{T}(H, M))$.

On the other hand, it is easy to see that every coherent functor arises as a functor $M \longmapsto \varphi(M)$ with $\varphi$ a pp formula. Indeed, given a coherent functor

$$
\mathcal{T}(K,-) \longrightarrow \mathcal{T}(H,-) \longrightarrow F \longrightarrow 0
$$

one can construct a triangle as above. Then the functor $F$ is the coherent functor $M \longrightarrow$ $\varphi(M)$ with $\varphi$ a pp-formula of the form $\alpha \mid v_{G}$.

Theorem 4.4 (first statement as [Zie] for $\operatorname{Mod} R$ ). Let $\mathcal{T}$ be a compactly generated triangulated category and suppose that $N \in(\alpha / \beta) \cap\left(\alpha^{\prime} / \beta^{\prime}\right)$ in Zsp $\mathcal{T}$. Then there is a basic open set $\left(\alpha^{\prime \prime} / \beta^{\prime \prime}\right)$ with $N \in\left(\alpha^{\prime \prime} / \beta^{\prime \prime}\right) \subseteq(\alpha / \beta) \cap\left(\alpha^{\prime} / \beta^{\prime}\right)$. Moreover, for any subset $(\alpha / \beta)$ in Zsp $\mathcal{T}$ there exists a pp formula $\delta$ such that $(\alpha / \beta)=(\delta)=\{N \in \operatorname{Zsp} \mathcal{T} \mid \delta(N) \neq 0\}$.

Proof. By Lemma 4.3 we can choose coherent functors $C$ and $D$ such that $\mathcal{O}(C)=(\alpha / \beta)$ and $\mathcal{O}(D)=\left(\alpha^{\prime} / \beta^{\prime}\right)$. By Corollary 4.2 there exists a basic open subset $\mathcal{O}(F)$ in $\mathcal{O}(C) \cap \mathcal{O}(D)$ containing $N$. Denote by $\delta$ a pp formula which corresponds to the coherent functor $F$. Then $N \in \mathcal{O}(F)=(\delta)$, as required.

Clearly, the Ziegler topology defined by pp formulas coincides with that defined by coherent functors. Below we shall freely use both definitions of the Ziegler topology on Zsp $\mathcal{T}$.

We say that $\mathcal{T}$ is Krull-Schmidt if every compact object is a (finite) coproduct of objects with local endomorphism ring. For example, the derived category $D(\Lambda)$ of an artin algebra $\Lambda$ is Krull-Schmidt because every perfect complex is endofinite and therefore is a coproduct of complexes with local endomorphism rings [Kr2, 1.2]. If $\mathcal{T}$ is Krull-Schmidt and $M$ is indecomposable compact, then its pure-injective hull $\widehat{M}$ is a point of Zsp $\mathcal{T}$ by Theorem 2.3. 
Proposition 4.5 (Herzog [He2]). Let $\mathcal{T}$ be Krull-Schmidt. The set of points having the form $\widehat{M}$ with $M$ a compact indecomposable is a dense subset of the Ziegler spectrum of $\mathcal{T}$.

Proof. Let $C \in \bmod \mathcal{T}^{c}$. There is a monomorphism in $\bmod \mathcal{T}^{c}$ of the form $\mu: C \longrightarrow H_{M}$ with $M$ a compact object. By assumption $H_{M} \simeq \coprod_{i=1}^{n} H_{M_{i}}$ with every $H_{M_{i}}$ a uniform object in $\operatorname{Mod} \mathcal{T}^{c}$. Then $\operatorname{Hom}\left(C, H_{M_{i}}\right) \neq 0$ for some $i \leq n$ and therefore $\widehat{M}_{i} \in \mathcal{O}(D C)$.

\section{Absolutely pure objects}

In this section we introduce the class of absolutely pure objects in $\mathcal{T}$. They share a number of important properties with absolutely pure modules in Mod $R$. We start with some definitions.

Let $\mathcal{R}$ be a family of compact objects in $\mathcal{T}$ closed under suspension. A map $X \longrightarrow Y$ in $\mathcal{T}$ is said to be an $\mathcal{R}$-monomorphism if the map $\mathcal{T}(C, X) \longrightarrow \mathcal{T}(C, Y)$ is a monomorphism for all $C$ in $\mathcal{R}$. An object $X$ in $\mathcal{T}$ is called $\mathcal{R}$-injective if every $\mathcal{R}$-monomorphism $\varphi: X \longrightarrow Y$ splits, i.e. there exists a map $\psi: Y \longrightarrow X$ such that $\psi \varphi=1$. For example, if $\mathcal{R}=\mathcal{T}^{c}$ we have the notions of pure monomorphism and pure-injective object. A triangle $X \longrightarrow Y \longrightarrow$ $Z \longrightarrow \Sigma X$ is called $\mathcal{R}$-exact if the sequence $0 \longrightarrow \mathcal{T}(C, X) \longrightarrow \mathcal{T}(C, Y) \longrightarrow \mathcal{T}(C, Z) \longrightarrow 0$ is an exact sequence for all $C \in \mathcal{R}$. We say that $X \in \mathcal{T}$ is $\mathcal{R}$-absolutely-pure if every $\mathcal{R}$ monomorphism $X \longrightarrow Y$ is a pure monomorphism. That is, if whenever $X \longrightarrow Y$ induces a monomorphism $\mathcal{T}(C, X) \longrightarrow \mathcal{T}(C, Y)$ for all $C \in \mathcal{R}$ it does so for all $C \in \mathcal{T}^{c}$. Clearly, a pureinjective object is $\mathcal{R}$-injective iff it is $\mathcal{R}$-absolutely pure. We also note that any coproduct of $\mathcal{R}$-absolutely pure objects is $\mathcal{R}$-absolutely pure.

Suppose $\mathcal{R}$ is a generating set. If there is no liklihood of confusion we refer to the corresponding $\mathcal{R}$-absolutely pure and $\mathcal{R}$-injective objects as well as to $\mathcal{R}$-monomorphisms and $\mathcal{R}$-exact triangles as absolutely pure and injective objects, and as monomorphisms and exact triangles respectively, omitting the prefix $\mathcal{R}$. We call a map $\varphi: X \longrightarrow Y$ an injective hull of $X$ if $\varphi$ is a monomorphism, $Y$ is injective and every endomorphism $\psi$ of $Y$ satisfying $\psi \varphi=\varphi$ is an isomorphism. Every object $X$ in $\mathcal{T}$ admits an injective hull $\varphi: X \longrightarrow Y$ and if $\varphi^{\prime}: X \longrightarrow Y^{\prime}$ is another injective hull, then there exists an isomorphism $\psi: Y \longrightarrow Y^{\prime}$ such that $\varphi^{\prime}=\psi \varphi[\mathrm{GaPr}]$. Therefore, an object $X$ is absolutely pure iff any monomorphism $\varphi: X \longrightarrow Y$ with $Y$ injective is a pure monomorphism.

Below we shall work with monogenic triangulated categories. If $X$ is a generator of $\mathcal{T}$ we consider the generating set $\mathcal{R}=\left\{\Sigma^{n} X\right\}_{n \in \mathbf{Z}}$. Let $S$ denote the $\mathbf{Z}$-graded ring $\mathcal{T}(X, X)_{*}=$ $\bigoplus_{n \in \mathbf{Z}} \mathcal{T}\left(\Sigma^{n} X, X\right)$. We retain this notation throughout the section. If $E$ is an injective $S$-module, then the functor

$$
\operatorname{Hom}_{S}\left(\mathcal{T}(X,-)_{*}, E\right): \mathcal{T} \longrightarrow \mathbf{A b}
$$

takes triangles to exact sequences and it takes direct sums to products. Brown's Representability Theorem in triangulated categories [Ne2] implies that there exists a representing object $\Gamma_{E}$ and a natural isomorphism

$$
\operatorname{Hom}_{S}\left(\mathcal{T}(X, Y)_{*}, E\right) \simeq \mathcal{T}\left(Y, \Gamma_{E}\right)
$$

for $Y \in \mathcal{T}$. The assignment $E \longrightarrow \Gamma_{E}$ yields a fully faithful functor

$$
\Gamma: \operatorname{lnj} S \longrightarrow \mathcal{T} .
$$

This $\Gamma$ identifies injective $S$-modules $\operatorname{Inj} S$ and $(\mathcal{R}$-)injective objects $\operatorname{Inj} \mathcal{T}$ in $\mathcal{T}$ (see [GaPr]). 
Example. (1) Let $\mathcal{R}=\{R[n]\}_{n \in \mathbf{Z}}$ be the generating set in $D(R)$, where $R[n]$ is the complex concentrated in the $-n$th degree. Since

$$
H^{n}(X)=D(R)(R[-n], X)
$$

for any complex $X$, we see that $X \longrightarrow Y$ is a $(\mathcal{R}$-)monomorphism in $D(R)$ iff the induced map of cohomology groups $H^{n}(X) \longrightarrow H^{n}(Y)$ is a monomorphism for all $n \in \mathbf{Z}$. A complex $X$ is injective iff it is a complex of the form $\coprod_{n \in \mathbf{Z}} Q^{n}[-n]$ with the $Q^{n}$ injective $R$-modules. An injective hull of a complex $X$ is constructed as follows. We take injective hulls $H^{n}(X) \longrightarrow Q^{n}$ of all cohomology groups of $X$. Then there exists a monomorphism $X \longrightarrow \coprod_{n \in \mathbf{Z}} Q^{n}[-n]$ which is an injective hull as well (see [GaPr] for details).

(2) A map $M \longrightarrow N$ in the stable category $\underline{\operatorname{Mod}} \Lambda$ of a QF-ring $\Lambda$ is a monomorphism (with respect to the generating set described before) iff the induced map

$$
\widehat{\operatorname{Ext}}_{\Lambda}^{n}(S, M) \longrightarrow \widehat{\operatorname{Ext}}_{\Lambda}^{n}(S, N)
$$

is a monomorphism for all simple modules $S$ and $n \in \mathbf{Z}$.

(3) Let $X$ be a spectrum in $\mathrm{Ho}(\mathcal{S})$ and let $\pi_{n}(X)=\operatorname{Ho}(\mathcal{S})\left(S^{n}, X\right)$ denote its stable homotopy groups. A morphism of spectra $X \longrightarrow Y$ is a monomorphism iff the induced map $\pi_{n}(X) \longrightarrow \pi_{n}(Y)$ is a monomorphism for all $n \in \mathbf{Z}$.

A result originally proved by Eklof and Sabbagh for module categories [ES] is the following.

Theorem 5.1. Let $\mathcal{T}$ be a monogenic triangulated category with a generator $X$. Then the class Abs $\mathcal{T}$ of absolutely pure objects of $\mathcal{T}$ is definable iff the ring $S$ is right coherent.

Proof. The ring $S$ is right coherent iff the set $\operatorname{Sp} \mathcal{T}$ of indecomposable injective objects in $\mathcal{T}$ is a closed subset in Zsp $\mathcal{T}$ [GaPr]. Since every object $X$ of $\mathcal{T}$ admits a monomorphism $X \longrightarrow \prod_{i} E_{i}$ with $E_{i} \in \operatorname{Sp} \mathcal{T}$ (see $[\mathrm{GaPr}]$ ), our statement follows from Theorem 4.1.

Below we describe absolutely pure complexes in the derived category $D(R)$. The generator $X$ we take to be $R[0]$ as above. So $S=\mathcal{T}(X, X)_{*} \simeq R$. Let $\mathcal{P}$ be the category of perfect complexes (=compact objects) in $D(R)$ and let $H: D(R) \longrightarrow$ Mod $\mathcal{P}$ be the functor sending a complex $X$ to the object $\left.D(R)(-, X)\right|_{\mathcal{P}}$. We say that two complexes $X$ and $Y$ are homologically isomorphic and write $X \simeq_{\text {hom }} Y$ if the functors $H_{X}$ and $H_{Y}$ are isomorphic in $\operatorname{Mod} \mathcal{P}$.

Theorem 5.2. All cohomology groups $H^{n}(X), n \in \mathbf{Z}$, of an absolutely pure complex $X \in$ $D(R)$ are absolutely pure modules. If, moreover, $R$ is a right coherent ring, then the following are equivalent:

(1) $X$ is absolutely pure;

(2) $X \simeq_{\text {hom }} \coprod_{n \in \mathbf{Z}} Y^{n}[-n]$ with the $Y^{n}$ absolutely pure modules.

The equivalent conditions (1)-(2) hold iff $X$ is homologically isomorphic to the coproduct of its cohomology groups $\coprod_{n \in \mathbf{Z}} H^{n}(X)[-n]$ and each $H^{n}(X)$ is an absolutely pure module.

Let $X$ be an absolutely pure complex and let $\varphi: X \longrightarrow Q=\coprod_{n \in \mathbf{Z}} Q^{n}[-n]$ be its injective hull. Each $Q^{n}$ is an injective hull of the $n$th cohomology group $H^{n}(X)$. Since $\varphi$ is a pure monomorphism, each $H^{n}(X), n \in \mathbf{Z}$, is an absolutely pure submodule of $Q^{n}$ by Lemma 2.4, and hence is itself absolutely pure. 
To prove the remainder of the theorem we need some preparation.

Recall that a Serre subcategory $\mathcal{S}$ of $\operatorname{Mod} \mathcal{P}$ is localizing if it is closed under taking direct limits. Equivalently, the inclusion functor $j: \mathcal{S} \longrightarrow \operatorname{Mod} \mathcal{P}$ admits the right adjoint $t=t_{\mathcal{S}}: \operatorname{Mod} \mathcal{P} \longrightarrow \mathcal{S}$ which takes every object $X \in \operatorname{Mod} \mathcal{P}$ to the maximal subobject $t(X)$ of $X$ belonging to $\mathcal{S}$. The functor $t$ we call the torsion functor. An object $C$ of $\operatorname{Mod} \mathcal{P}$ is said to be $\mathcal{S}$-torsionfree if $t(C)=0$. Given a localizing subcategory $\mathcal{S}$ of $\operatorname{Mod} \mathcal{P}$ the quotient category, $\operatorname{Mod} \mathcal{P} / \mathcal{S}$, is the full subcategory on those $C \in \operatorname{Mod} \mathcal{P}$ such that $\operatorname{Hom}(\mathcal{S}, C)=\operatorname{Ext}^{1}(\mathcal{S}, C)=0$. The objects from $\operatorname{Mod} \mathcal{P} / \mathcal{S}$ we call $\mathcal{S}$-closed objects. For any $C \in \operatorname{Mod} \mathcal{P}$ there exists a canonical exact sequence

$$
0 \longrightarrow A^{\prime} \longrightarrow C \stackrel{\lambda_{C}}{\longrightarrow} C_{\mathcal{S}} \longrightarrow A^{\prime \prime} \longrightarrow 0
$$

with $A^{\prime}=t(C), A^{\prime \prime} \in \mathcal{S}$, and where $C_{\mathcal{S}} \in \operatorname{Mod} \mathcal{P} / \mathcal{S}$ is the maximal essential extension of $\widetilde{C}=C / t(C)$ such that $C_{\mathcal{S}} / \widetilde{C} \in \mathcal{S}$. The object $C_{\mathcal{S}}$ is unique up to isomorphism. and the morphism $\lambda_{C}: C \longrightarrow C_{\mathcal{S}}$ is called the $\mathcal{S}$-hull of $C$. The $\mathcal{S}$-hull has the property that given any morphism $\alpha: C \longrightarrow W$ with $W$ an $\mathcal{S}$-closed object, there is a unique morphism $\alpha_{\mathcal{S}}: C_{\mathcal{S}} \longrightarrow W$ such that $\alpha_{\mathcal{S}} \lambda_{C}=\alpha$.

Thus the inclusion functor $i: \operatorname{Mod} \mathcal{P} / \mathcal{S} \longrightarrow \operatorname{Mod} \mathcal{P}$ has the left adjoint localizing functor $(-)_{\mathcal{S}}: \operatorname{Mod} \mathcal{P} \longrightarrow \operatorname{Mod} \mathcal{P} / \mathcal{S}$ which takes each $C \in \operatorname{Mod} \mathcal{P}$ to $C_{\mathcal{S}} \in \operatorname{Mod} \mathcal{P} / \mathcal{S}$. There is an isomorphism

$$
\operatorname{Hom}(X, Y) \simeq \operatorname{Hom}\left(X_{\mathcal{S}}, Y\right)
$$

for all $X \in \operatorname{Mod} \mathcal{P}$ and $Y \in \operatorname{Mod} \mathcal{P} / \mathcal{S}$. The functor $(-)_{\mathcal{S}}$ is exact. We say that $\mathcal{S}$ is of finite type if the inclusion functor $i: \operatorname{Mod} \mathcal{P} / \mathcal{S} \longrightarrow \operatorname{Mod} \mathcal{P}$ preserves direct limits.

Let $\operatorname{Mod} \mathcal{R}$ denote the category of contravariant functors defined on $\mathcal{R}=\{R[n]\}_{n \in \mathbf{Z}}$. Then $\operatorname{Mod} \mathcal{R}$ is equivalent to the quotient category $\operatorname{Mod} \mathcal{P} / \mathcal{S}$ with $\mathcal{S}$ the localizing subcategory $\{F \in \operatorname{Mod} \mathcal{P} \mid F(\mathcal{R})=0\}[\mathrm{G}]$.

Since

the functor

$$
D(R)(R[n], R[m])=\left\{\begin{array}{ccc}
R, & n=m \\
0, & n \neq & m
\end{array}\right.
$$

$$
\begin{aligned}
\operatorname{Mod} \mathcal{R} & \longrightarrow(\operatorname{Mod} R)^{\mathbf{Z}}=\prod_{\mathbf{Z}} \operatorname{Mod} R \\
M \longmapsto & \left(M^{n}\right)_{n \in \mathbf{Z}}, \quad M^{n}=M(R[n])
\end{aligned}
$$

is an equivalence of categories. Below we shall consider this equivalence as an identification.

The image of a functor $F$ under the localization functor $(-)_{\mathcal{S}}: \operatorname{Mod} \mathcal{P} \longrightarrow \operatorname{Mod} \mathcal{P} / \mathcal{S}$ is isomorphic to the $\mathcal{S}$-localization of the functor $H_{\widetilde{F}}$ where $\widetilde{F}$ is the complex with zero differential $(F(R[n]), 0)$. We also notice that for any complex , $\left(M^{n}, 0\right)$, with zero differential the following relations hold in $D(R)$ :

$$
M=\coprod_{n \in \mathbf{Z}} M^{n}[-n]=\prod_{n \in \mathbf{Z}} M^{n}[-n],
$$

where $M^{n}[-n]$ is the complex with $M^{n}$ in the $n$th degree and zero in other degrees [GaPr]. It follows that an object of $\operatorname{Mod} \mathcal{P}$ is $\mathcal{S}$-closed injective iff it is isomorphic to a functor of the form $H_{Q}$, where $Q$ is a complex with zero differential $\left(Q^{n}, 0\right)$ whose components $Q^{n}$ are injective $R$-modules. (Note that an object of $(\operatorname{Mod} R)^{\mathbf{Z}}$ is injective iff each component if injective.) 
Lemma 5.3. Let $X$ be a complex and let $f: H_{X} \longrightarrow M$ be a morphism from $H_{X}$ to an absolutely pure object $M$ in $\operatorname{Mod} \mathcal{P}$. If $f(R[n]): H^{-n}(X) \longrightarrow M(R[n])$ is an isomorphism of $R$-modules for all $n \in \mathbf{Z}$, then $f$ is an isomorphism.

Proof. Since both $H_{X}$ and $M$ are cohomological functors on $\mathcal{P}$, it follows by standard arguments that $f$ is still bijective evaluated on any perfect complex, since $\mathcal{P}$ is the thick subcategory generated by $R$. This means that $f$ is an isomorphism.

Proof of Theorem 5.2. Suppose that $R$ is right coherent. Then the localizing subcategory $\mathcal{S}$ is of finite type [GaPr]. Let $X$ be an absolutely pure complex and let $\varphi: X \longrightarrow Q$ be its injective hull. The object $H_{Q}$ is $\mathcal{S}$-closed injective in $\operatorname{Mod} \mathcal{P}$. Since $\varphi$ is a pure monomorphism, the object $H_{X}$ is a subobject of $H_{Q}$, and hence is $\mathcal{S}$-torsionfree. Moreover, $H_{X}$ is absolutely pure in $\operatorname{Mod} \mathcal{P}$. Since $\mathcal{S}$ is of finite type, it follows from $[\mathrm{He} 2,3.10]$ that $H_{X}$ is an $\mathcal{S}$-closed object. We claim that $H_{X}$ is isomorphic to the object $H_{\left(Y^{n}, 0\right)}$ with $Y^{n}=H^{n}(X)$. We have already seen that each $Y^{n}$ is an absolutely pure module.

Indeed, the $\mathcal{S}$-localization both of the object $H_{\left(Y^{n}, 0\right)}$ and of the object $H_{X}$ is $\left(H_{\left(Y^{n}, 0\right)}\right)_{S}$. Since $H_{X}$ is $\mathcal{S}$-closed, it is isomorphic to $\left(H_{\left(Y^{n}, 0\right)}\right)_{\mathcal{S}}$. By $(2)$ we obtain an exact sequence

$$
0 \longrightarrow A^{\prime} \longrightarrow H_{\left(Y^{n}, 0\right)} \stackrel{\lambda}{\longrightarrow} H_{X} \longrightarrow A^{\prime \prime} \longrightarrow 0
$$

with $A^{\prime}$ and $A^{\prime \prime}$ in $\mathcal{S}$. The map $\lambda(R[n])$ is an isomorphism for all $n \in \mathbf{Z}$. From Lemma 5.3 it follows that $\lambda$ is an isomorphism in $\operatorname{Mod} \mathcal{P}$. Thus $X$ is homologically isomorphic to the complex $\coprod_{n \in \mathbf{Z}} Y^{n}[-n]$, and the implication (1) $\Longrightarrow(2)$ follows.

$(2) \Longrightarrow(1)$. A sequence

$$
0 \longrightarrow L \longrightarrow M \longrightarrow N \longrightarrow 0
$$

of $\operatorname{Mod} \mathcal{P}$ is pure-exact if the sequence

$$
0 \longrightarrow \operatorname{Hom}(F, L) \longrightarrow \operatorname{Hom}(F, M) \longrightarrow \operatorname{Hom}(F, N) \longrightarrow 0
$$

is exact for each finitely presented $F \in \bmod \mathcal{P}$. Equivalently, the latter is a direct limit of split exact sequences.

Let $I$ denote the composed functor $\operatorname{Mod} \mathcal{R} \stackrel{\sim}{\longrightarrow} \operatorname{Mod} \mathcal{P} / \mathcal{S} \stackrel{i}{\longrightarrow} \operatorname{Mod} \mathcal{P}$. Since $\mathcal{S}$ is of finite type, $I$ preserves direct limits. Let

$$
\varepsilon: 0 \longrightarrow X_{1} \longrightarrow X_{2} \longrightarrow X_{3} \longrightarrow 0
$$

be a pure-exact sequence in $\operatorname{Mod} \mathcal{R}$. This sequence is a direct limit $\varepsilon=\underline{\lim } \varepsilon_{k}$ of split exact sequences

$$
\varepsilon_{k}: 0 \longrightarrow Y_{1}^{k} \longrightarrow Y_{2}^{k} \longrightarrow Y_{3}^{k} \longrightarrow 0 .
$$

Hence $I(\varepsilon)=\lim _{\longrightarrow} I\left(\varepsilon_{k}\right)$ is a direct limit of split exact sequences $I\left(\varepsilon_{k}\right)$ and is therefore pureexact in $\operatorname{Mod} \mathcal{P}$.

Let us consider an object $M=\left(M^{n}\right)_{n \in \mathbf{Z}}$ of $\operatorname{Mod} \mathcal{R}$ with the $M^{n}$ absolutely pure modules. Then it is an absolutely pure object in $\operatorname{Mod} \mathcal{R}$. Consider the pure-exact sequence in $\operatorname{Mod} \mathcal{R}$

$$
\varepsilon: 0 \longrightarrow M \longrightarrow Q \longrightarrow Z \longrightarrow 0
$$

with $Q=\left(Q^{n}\right)_{n \in \mathbf{Z}}$ being an injective hull of $M$ and $Z=\left(Z^{n}=Q^{n} / M^{n}\right)_{n \in \mathbf{Z}}$. It follows that the sequence

$$
I(\varepsilon): 0 \longrightarrow I(M) \longrightarrow I(Q) \longrightarrow I(Z) \longrightarrow 0
$$


is pure-exact in $\operatorname{Mod} \mathcal{P}$. Since $Q$ is an injective object, it follows that $I(Q)=H_{\left(Q^{n}, 0\right)}$. In particular $I(Q)$ is an absolutely pure object in $\operatorname{Mod} \mathcal{P}$. So $I(M)$, being a pure subobject, also is absolutely pure in $\operatorname{Mod} \mathcal{P}$. As above we see that $H_{\left(M^{n}, 0\right)}$ is isomorphic to $I(M)=$ $\left(H_{\left(M^{n}, 0\right)}\right)_{s}$. It follows that $H_{\left(M^{n}, 0\right)}$ is a subobject of $H_{\left(Q^{n}, 0\right)}$ and therefore the complex $M=\left(M^{n}, 0\right)=\bigsqcup_{n \in \mathbf{Z}} M^{n}[-n]$ is absolutely pure in $D(R)$.

It remains to check that any complex $X$ homologically isomorphic to an absolutely pure complex $Y$ is absolutely pure. Indeed, let $\varphi: Y \longrightarrow Q$ be an injective hull of $Y$ (it exists by $[\mathrm{GaPr}])$. Then $\varphi$ is a pure monomorphism. The composed map

$$
H_{X} \stackrel{\sim}{\longrightarrow} H_{Y} \stackrel{H_{\varphi}}{\longrightarrow} H_{Q}
$$

is represented by a map $\psi: X \longrightarrow Q[\mathrm{Kr} 3,1.8]$. This $\psi$ is plainly a pure monomorphism. Therefore $X$ is absolutely pure. The proof is complete.

Corollary 5.4. Let $R$ be a right coherent ring. A complex $X$ is absolutely pure iff all its cohomology groups $H^{n}(X), n \in \mathbf{Z}$, are absolutely pure $R$-modules.

Proof. By the preceding theorem all cohomology groups $H^{n}(X), n \in \mathbf{Z}$, of an absolutely pure complex $X$ are absolutely pure modules.

For the converse note that the proof of the implication $(2) \Longrightarrow(1)$ of the preceding theorem shows that the $\mathcal{S}$-localization of the object $H_{X}$ is isomorphic to $H_{\left(Y^{n}, 0\right)}$ where $Y^{n}=H^{n}(X)$. Then the $\mathcal{S}$-hull $\lambda: H_{X} \longrightarrow H_{\left(Y^{n}, 0\right)}$ is an isomorphism by Lemma 5.3. Thus $X$ is homologically equivalent to the absolutely pure complex $\bigsqcup_{n \in \mathbf{Z}} Y^{n}$. Theorem 5.2 then yields the claim.

\section{The Ziegler spectrum of QF-Rings and the stable module Category}

In the remaining sections we shall study the relationship between the Ziegler spectrum Zsp $R$ of a ring and Ziegler spectra of related triangulated categories. We start with some definitions.

The Ziegler spectrum Zsp $R$ of a ring $R$ is defined as follows. Let $\mathcal{C}_{R}=(R \bmod , \mathbf{A b})$ denote the locally coherent category of additive covariant functors defined on the category, $R$ mod, of finitely presented left $R$-modules with values in the category, $\mathbf{A b}$, of abelian groups. The functor

$$
? \otimes_{R}-: \operatorname{Mod} R \longrightarrow \mathcal{C}_{R}, \quad Q \longmapsto Q \otimes_{R}-,
$$

identifies the pure-injectives in $\operatorname{Mod} R$ with the injectives in $\mathcal{C}_{R}$. The collection of subsets

$$
\Omega(X)=\left\{Q \in \operatorname{Zsp} R \mid\left(X, Q \otimes_{R}-\right) \neq 0\right\},
$$

where $X \in \mathrm{fp}_{R}$ is a finitely presented object in $\mathcal{C}_{R}$, forms a basis of open subsets for the Ziegler topology on Zsp $R$ (see [Zie, He2, Kr1]). This topological space we call the Ziegler spectrum of the ring $R$.

This topological space can also be defined by using coherent functors. A functor $C$ : $\operatorname{Mod} R \longrightarrow \mathbf{A b}$ is coherent if there exists an exact sequence

$$
\left(M_{R},-\right) \longrightarrow\left(N_{R},-\right) \longrightarrow C \longrightarrow 0
$$

with $M$ and $N$ finitely presented. The collection of subsets

$$
\mathcal{O}(C)=\{Q \in \operatorname{Zsp} R \mid C(Q) \neq 0\}
$$


with $C$ a coherent functor forms a basis of open subsets for a topology on Zsp $R$ and this topological space coincides with the Ziegler spectrum defined above. An open subset $\mathcal{O}$ in Zsp $R$ is quasi-compact iff it is one of the basic open subsets $\mathcal{O}(C)$ with $C$ a coherent functor.

Let $\Lambda$ be a QF-ring. The relationship between the Ziegler spectra Zsp $\Lambda$ and Zsp Mod $\Lambda$ is easily established. For the most part we follow the paper of Benson and Krause [BK].

The Ziegler spectrum Zsp $\Lambda$ is the disjoint union $\mathcal{O} \cup Z s p \Lambda$ of the (finite) subset of indecomposable projectives (=injectives) $\mathcal{O}$ and the subset $\overline{Z s p} \Lambda$ of nonprojective elements of $Z$ sp $\Lambda$. Since every indecomposable projective has finite endolength each point $P \in \mathcal{O}$ is closed. Such a point, being finitely presented and the injective hull of a simple module, is also open (e.g. $[\mathrm{PrPu}, 3.7])$. Therefore both $\mathcal{O}$ and $\underline{\mathrm{Zsp} \Lambda}$ are clopen in $Z \mathrm{sp} \Lambda$.

The Ziegler spectrum Zsp $\underline{\operatorname{Mod}} \Lambda$ may be identified with $\underline{Z s p} \Lambda$ because by [Kr3] it consists of nonprojective pure-injective indecomposable $\Lambda$-modules. Let us show that the Ziegler topology on Zsp Mod $\Lambda$ coincides with the subspace topology induced from Zsp $\Lambda$.

Given a coherent functor $C: \operatorname{Mod} \Lambda \longrightarrow \mathbf{A b}$, it follows that $\mathcal{O}(C) \backslash \mathcal{O}$ is a quasi-compact subset of $\operatorname{Zsp} \Lambda$ since $\mathcal{O}(C)$ is quasi-compact and $\mathcal{O}$ is finite clopen in $\operatorname{Zsp} \Lambda$. So there exists a coherent functor $D$ such that $\mathcal{O}(D)=\mathcal{O}(C) \backslash \mathcal{O}$. By construction, $D$ vanishes on every projective module. One can show that $D$ has a presentation of the form

$$
\underline{\operatorname{Hom}}_{\Lambda}(Y,-) \longrightarrow \underline{\operatorname{Hom}}_{\Lambda}(X,-) \longrightarrow D \longrightarrow 0
$$

with $X$ and $Y$ finitely generated. So $D$ is a coherent functor on $\underline{\operatorname{Mod}} \Lambda$ and we see that $\mathcal{O}(D)=\mathcal{O}(C) \cap Z \operatorname{sp} \underline{\operatorname{Mod}} \Lambda$.

On the other hand, each coherent functor (3) gives a coherent functor $C=D \circ \pi$ vanishing on projectives where $\pi: \operatorname{Mod} \Lambda \longrightarrow \underline{\operatorname{Mod}} \Lambda$ is the natural functor. Then $\mathcal{O}(D)=\mathcal{O}(C) \cap$ Zsp $\underline{\operatorname{Mod}} \Lambda$.

Thus the following statement is true.

Proposition 6.1. The Ziegler spectrum Zsp $\Lambda$ of a QF-ring $\Lambda$ is homeomorphic to the disjoint union

$$
\mathrm{Zsp} \Lambda \simeq \mathcal{O} \bigsqcup \mathrm{Zsp} \underline{\operatorname{Mod} \Lambda}
$$

of the finite clopen subset $\mathcal{O}$ consisting of indecomposable projectives and the Ziegler spectrum, Zsp $\underline{\operatorname{Mod}} \Lambda$, of the stable category $\underline{\operatorname{Mod} \Lambda}$.

\section{The Ziegler spectra Zsp $R$ And Zsp $D(R)$}

In this section we study the relationship between the Ziegler spectra Zsp $R$ and Zsp $D(R)$.

Let $I_{n}: \operatorname{Mod} R \longrightarrow D(R)$ be the functor that takes a module $M$ to the complex $M[-n]$ concentrated in the $n$th degree. The functor $I_{n}$ gives an equivalence between $\operatorname{Mod} R$ and the full subcategory of $D(R)$ consisting of those complexes $X$ such that $H^{i}(X)=0$ for all $i \neq n$. On the other hand, let $H^{n}: D(R) \longrightarrow \operatorname{Mod} R$ denote the functor sending a complex $X$ to its $n$th cohomology group $H^{n}(X)$.

Proposition 7.1. Let $X$ be a pure-injective complex in $D(R)$. Then each cohomology group $H^{n}(X)$ is a pure-injective module. On the other hand, every complex $Q[-n]$ with $Q$ a pure-injective module is pure-injective in $D(R)$.

Proof. A module $M$ is pure-injective iff for every set $I$ the summation map $M^{(I)} \longrightarrow M$ factors through the canonical map $M^{(I)} \longrightarrow M^{I}$. Since the cohomology functor $H^{n}$ preserves 
both products and coproducts, it sends pure-injective complexes to pure-injective modules by Theorem 2.1 .

On the other hand, the functor $I_{n}$ commutes with products and coproducts, as one easily sees. By Theorem 2.1 we deduce that $I_{n}$ transfers pure-injective modules to pure-injective complexes.

A compactly triangulated category $\mathcal{T}$ is pure-semisimple if every object of $\mathcal{T}$ is pureinjective.

Corollary 7.2. If the derived category $D(R)$ of $\operatorname{Mod} R$ is pure-semisimple then $R$ is a right pure-semisimple ring.

Remark. The converse is not true. For example, let $\Lambda$ be a representation finite and of infinite global dimension artin algebra. Then the derived category $D(\Lambda)$ is not puresemisimple [Bel1, 12.16].

As a consequence of Proposition 7.1 we get that $I_{n}$ maps the set Zsp $R$ of indecomposable pure-injective modules to indecomposable pure-injective complexes of $\operatorname{Zsp} D(R)$. We also denote this map by $I_{n}$. Its image $\{Q[-n] \mid Q \in Z$ sp $R\}$ will be denoted by $Z_{s_{n}} R$. We may view this map $I_{n}$ as an identification.

Theorem 7.3. The following statements are true for a ring $R$.

(1) Each $\mathrm{Zsp}_{n} R, n \in \mathbf{Z}$, is a closed subset in Zsp $D(R)$;

(2) If $R$ is right coherent and every finitely presented module is of finite projective dimension, then $I_{n}$ induces a homeomorphism between $Z s p R$ and each of its images, $\mathrm{Zsp}_{n} R$, in $\mathrm{Zsp} D(R)$;

(3) The disjoint union $\bigcup_{n \in \mathbf{Z}} Z_{s p_{n}} R$ is a closed subset in $Z s p D(R)$. Its open complement $X$ consists of the indecomposable pure-injective complexes having at least two nonzero cohomology groups. Thus the following relation between the Ziegler spectra Zsp $R$ and Zsp $D(R)$ holds:

$$
\text { Zsp } D(R)=X \cup \bigcup_{n \in \mathbf{Z}} \mathrm{Zsp}_{n} R .
$$

Proof. (1). For each $i \in \mathbf{Z}$, we consider the closed set

$$
\left[v_{i}=v_{i}\right]=\left(v_{i}=v_{i}\right)^{c}=\left\{X \in \operatorname{Zsp} D(R) \mid H^{i}(X)=0\right\},
$$

where $v_{i}$ denotes a variable of sort $R[-i]$. The intersection $\mathcal{U}=\bigcap_{i \neq n}\left[v_{i}=v_{i}\right]$ is a closed subset in $\operatorname{Zsp} D(R)$. Clearly, a complex $X$ is in $\mathcal{U}$ iff $X \simeq H^{n}(X)[-n]$. By the preceding proposition we see that $\mathcal{U}=\{Q[-n] \mid Q \in Z$ sp $R\}$. Hence $Z_{s p} R$ is a closed subset in Zsp $D(R)$.

(2). Suppose now that the ring $R$ is right coherent and that every finitely presented module is of finite projective dimension. Then every complex $M[-n]$ with $M$ a finitely presented module is a perfect complex in $D(R)$. We must show that the Zigler topology on $\operatorname{Zsp}_{n} R=\{Q[-n] \mid Q \in \mathrm{Zsp} R\}$ coincides with the subspace topology induced from $\operatorname{Zsp} D(R)$.

Let $C: \operatorname{Mod} R \longrightarrow \mathbf{A b}$ be a coherent functor and let $\Omega(C)=\{Q \in \operatorname{Zsp} R \mid C(Q) \neq 0\}$ be a basic open subset in Zsp $R$. There exists an exact sequence

$$
\left(M_{R},-\right) \stackrel{(\alpha,-)}{\longrightarrow}\left(L_{R},-\right) \longrightarrow C \longrightarrow 0
$$


with $M$ and $L$ finitely presented and $\alpha: L \longrightarrow M$ a morphism. We consider the perfect complexes $M[-n]$ and $L[-n]$ and the coherent functor $F: D(R) \longrightarrow$ Ab defined by the following exact sequence where $\alpha^{*}$ is induced by $\alpha$ :

$$
(M[-n],-) \stackrel{\alpha^{*}}{\longrightarrow}(L[-n],-) \longrightarrow F \longrightarrow 0 .
$$

Then $\mathcal{O}(F)=\{X \in \operatorname{Zsp} D(R) \mid F(X) \neq 0\}$ is a basic open subset in Zsp $D(R)$ and $\Omega(C)=$ $\mathcal{O}(F) \cap \mathrm{Zsp}_{n} R$.

Conversely, suppose that $F: D(R) \longrightarrow \mathbf{A b}$ is a coherent functor. We claim that the restriction, $\widetilde{F}=\left.F\right|_{\operatorname{Mod} R}$, of $F$ to $\operatorname{Mod} R$ is a coherent functor $\operatorname{Mod} R \longrightarrow \mathbf{A b}$ (here $\operatorname{Mod} R$ is viewed as the full subcategory of complexes $M[-n]$ concentrated in the $n$th degree). To show this we use the fact that a functor from $\operatorname{Mod} R$ to $\mathbf{A b}$ is coherent iff it commutes with products and direct limits. Obviously, $\widetilde{F}$ commutes with products. Let $\mathbf{y}: \operatorname{Mod} R \longrightarrow$ $\operatorname{Mod} \mathcal{P}$, where $\mathcal{P}$ is the category of perfect (=compact) complexes in $D(R)$, be the functor that sends a module $M$ to the functor $\left.D(R)(-, M[-n])\right|_{\mathcal{P}}$. The functor $\mathbf{y}$ factors through $I_{n}$. By [CKN, 1.3] y commutes with direct limits. Let $\left\{M_{i}\right\}_{i \in I}$ be a direct system of modules with limit $M$. The complex $M[-n]$ is the homology colimit, in the sense of [Kr4], of the complexes $M_{i}[-n]$. By $[\mathrm{Kr} 4,5.1] F$ respects homology colimits. It then follows that $\widetilde{F}$ preserves direct limits. So $\widetilde{F}$ is a coherent functor and hence $\mathcal{O}(F) \cap \mathrm{Zsp}_{n} R=\Omega(\widetilde{F})$.

(3). Given $m, n \in \mathbf{Z}$ let $\mathcal{O}_{m n}$ denote the open subset $\left(v_{m}=v_{m}\right) \cap\left(v_{n}=v_{n}\right)$. By definition, a complex $X$ belongs to $\mathcal{O}_{m n}$ iff the cohomology groups $H^{m}(X)$ and $H^{n}(X)$ are non-zero. It is clear that

$$
X=\operatorname{Zsp} D(R) \backslash \bigcup_{n \in \mathbf{Z}} \operatorname{Zsp}_{n} R=\bigcup_{m \neq n} \mathcal{O}_{m n},
$$

as required.

Remark. The disjoint union $\bigcup_{n \in \mathbf{Z}} Z_{s p} R$ is a proper subset of $Z s p D(R)$ in general. The following example shows this.

Consider the finite-dimensional algebra $R$, over an algebraically closed field $k$, which is the path algebra of the quiver $A_{10}$

$$
1 \stackrel{\alpha_{1}}{\longrightarrow} 2 \stackrel{\alpha_{2}}{\longrightarrow} 3 \longrightarrow \cdots \longrightarrow 9 \stackrel{\alpha_{9}}{\longrightarrow} 10
$$

with the relation $\alpha_{8} \alpha_{7} \cdots \alpha_{1}=0$. The algebra $R$ is a quotient of $k\left[A_{10}\right]$ and thus is of finite representation type.

Let $S$ be the path algebra of the quiver

$$
\begin{aligned}
2 \rightarrow \cdots \rightarrow & 8 \\
& \rightarrow \quad 9 \rightarrow 10 \\
& 1
\end{aligned}
$$

So $S$ is of infinite representation type. Then the derived categories $D(R)$ and $D(S)$ are triangle equivalent $[\mathrm{CKN}]$. This equivalence induces a homeomorphism of Ziegler spectra, Zsp $D(R) \simeq Z \operatorname{sp} D(S)$. Since we have finitely many indecomposable pure-injective $R$ modules, we see that $\bigcup_{n \in \mathbf{Z}} Z \mathrm{sp}_{n} R$ is a countable set. On the other hand, suppose that $k$ is an uncountable field. Then Zsp $S$ is uncountable and therefore $Z \operatorname{sp} D(R) \simeq Z \operatorname{sp} D(S) \supset \operatorname{Zsp}_{0} S$ is uncountable as well.

By a theorem of Herzog [He1] there is an isomorphism between the topologies, that is the algebras of open sets, of the Ziegler spectra Zsp $R$ and Zsp $R^{\mathrm{op}}$. Similarly, we can construct 
an isomorphism between the algebras of open sets of the Ziegler spectra Zsp $D(R)$ and Zsp $D\left(R^{\mathrm{op}}\right)$. We do this in the following way.

Consider two compactly generated triangulated categories $\mathcal{S}$ and $\mathcal{T}$. Suppose that there is a duality $D: \mathcal{S}^{c} \longrightarrow \mathcal{T}^{c}$ between the corresponding subcategories of compact objects $\mathcal{S}^{c}$ and $\mathcal{T}^{c}$. This induces an equivalence of categories

$$
\Theta: \bmod \mathcal{S}^{c} \stackrel{\sim}{\longrightarrow} \mathcal{T}^{c} \bmod ,
$$

where $\mathcal{T}^{c}$ mod is the abelian subcategory of finitely presented objects of the category, $\left(\mathcal{T}^{c}, \mathbf{A b}\right)$, of additive covariant functors on $\mathcal{T}^{c}$.

Let $\Delta$ denote the functor sending an object $M$ of $\mathcal{T}^{c} \bmod$ to an object of $\bmod \mathcal{T}^{c}$ defined by

$$
\Delta(M)(X)=\operatorname{Hom}\left(M, H^{X}\right)
$$

with $X \in \mathcal{T}^{c}$ and $H^{X}=\left.\mathcal{T}(X,-)\right|_{\mathcal{T}^{c}}$. Then $\Delta$ puts the categories $\mathcal{T}^{c} \bmod$ and $\bmod \mathcal{T}^{c}$ in duality. Indeed, the categories of flat and absolutely pure $\mathcal{T}^{c}$-modules coincide [Bel2, $\left.\mathrm{Kr} 3\right]$. Therefore $\Delta$ is a duality by $[\mathrm{G}, 8.14]$ (every small triangulated category is weakly quasiFrobenius in the sense of $[\mathrm{G}]$ ). It follows that the composite functor $\Gamma=\Delta \circ \Theta$ yields a duality between the categories $\bmod \mathcal{S}^{c}$ and $\bmod \mathcal{T}^{c}$.

Let $\mathcal{L}$ be a Serre subcategory in $\bmod \mathcal{S}^{c}$. It is clear that the subcategory

$$
\Gamma \mathcal{L}=\left\{\Gamma(M) \mid M \in \bmod \mathcal{S}^{c}\right\}
$$

of $\bmod \mathcal{T}^{c}$ is also Serre and that the restriction to $\mathcal{L}$ of $\Gamma$ gives a duality $\Gamma: \mathcal{L} \longrightarrow \Gamma \mathcal{L}$. Moreover, the map $\mathcal{O}(\mathcal{L}) \longrightarrow \mathcal{O}(\Gamma \mathcal{L})$ induced on the open subsets of the Ziegler spectra Zsp $\mathcal{S}$ and Zsp $\mathcal{T}$ is an inclusion-preserving bijection (cf. [He2, 3.8]). We summarize all of this as follows.

Theorem 7.4 (cf. [He2]). Suppose that there is a duality between $\mathcal{S}^{c}$ and $\mathcal{T}^{c}$. Then the functor $\Gamma$ defined above yields an inclusion-preserving bijective correspondence between the Serre subcategories of $\bmod \mathcal{S}^{c}$ and those of $\bmod \mathcal{T}^{c}$ given by $\mathcal{L} \longrightarrow \Gamma \mathcal{L}$. The induced map $\mathcal{O}(\mathcal{L}) \longrightarrow \mathcal{O}(\Gamma \mathcal{L})$ is an isomorphism between the topologies, that is, the respective algebras of open sets, of the Ziegler spectra Zsp $\mathcal{S}$ and Zsp $\mathcal{T}$.

We apply the preceding theorem to compare the Ziegler spectra Zsp $D(R)$ and Zsp $D\left(R^{\mathrm{op}}\right)$. For this, we consider the equivalence

$$
(\operatorname{proj} R)^{\mathrm{op}} \longrightarrow \operatorname{proj} R^{\mathrm{op}}, \quad P \longmapsto D P=\operatorname{Hom}_{R}(P, R),
$$

which induces an equivalence

$$
D: \mathcal{H}^{b}(\operatorname{proj} R)^{\mathrm{op}} \longrightarrow \mathcal{H}^{b}\left(\operatorname{proj} R^{\mathrm{op}}\right) .
$$

Here $\mathcal{H}^{b}(\operatorname{proj} R)$ stands for the homotopy category of bounded complexes of objects in $\operatorname{proj} R$. The duality $D$ induces a duality between the categories of perfect (=compact) complexes $\mathcal{P}(R)$ and $\mathcal{P}\left(R^{\mathrm{op}}\right)$ in $D(R)$ and $D\left(R^{\mathrm{op}}\right)$ respectively, since $\mathcal{H}^{b}($ proj $R)$ and $\mathcal{P}(R)$ are naturally equivalent. By the above $D$ induces a duality

$$
\Gamma: \bmod \mathcal{P}(R) \longrightarrow \bmod \mathcal{P}\left(R^{\mathrm{op}}\right) .
$$

The preceding theorem implies the following. 
Corollary 7.5. Let $R$ be a ring. There is an inclusion-preserving bijective correspondence between the Serre subcategories of $\bmod \mathcal{P}(R)$ and those of $\bmod \mathcal{P}\left(R^{\mathrm{op}}\right)$ given by $\mathcal{L} \longrightarrow \Gamma \mathcal{L}$. The induced map $\mathcal{O}(\mathcal{L}) \longrightarrow \mathcal{O}(\Gamma \mathcal{L})$ is an isomorphism between the topologies, that is, the respective algebras of open sets, of the Ziegler spectra Zsp $D(R)$ and $Z s p D\left(R^{\mathrm{op}}\right)$.

\section{Zsp $D(R)$ OF HEREditary AND von Neumann REgular Rings}

We finish the paper by describing the Ziegler spectra $Z \operatorname{sp} D(R)$ for hereditary and von Neumann regular rings.

By a result of Neeman [Ne1], any complex $X$ in $D(R)$ over a right hereditary ring $R$ is isomorphic to the coproduct of its cohomology groups $\bigsqcup_{n \in \mathbf{Z}} H^{n}(X)[-n]$.

Theorem 8.1. Let $R$ be a right hereditary ring. A complex $X$ is pure-injective in $D(R)$ iff it is a complex of the form $\bigsqcup_{n \in \mathbf{Z}} Q^{n}[-n]$ with the $Q^{n}$ pure-injective $R$-modules. Moreover, the Ziegler spectrum of the derived category $D(R)$ is homeomorphic to the disjoint union

$$
\operatorname{Zsp} D(R) \simeq \bigsqcup_{n \in \mathbf{Z}} \mathrm{Zsp} R
$$

of countably many copies of Zsp $R$.

Proof. The first part of the theorem follows from Proposition 7.1 and the fact that a coproduct of the form $\amalg M^{n}[-n]$ is isomorphic to the product $\prod M^{n}[-n]$ (e.g. see [GaPr, 3.9]). Since every indecomposable pure-injective complex is of the form $Q[-n]$ for some $Q \in \operatorname{Zsp} R$, each subset $Z_{s p_{n}} R$ is the basic open $\left(v_{n}=v_{n}\right)$ in $Z$ sp $D(R)$, where $v_{n}$ is a variable of sort $R[-n]$. Every right hereditary ring is right coherent and every finitely presented module is of projective dimension at most one. It remains to apply Theorem 7.3.

The Ziegler spectrum Zsp $D(R)$ of a von Neumann regular ring is similarly characterized (see below). First we need the following result.

Theorem 8.2. For a ring $R$ the following are equivalent:

(1) $R$ is von Neumann regular;

(2) every pure-injective complex is injective;

(3) every complex is absolutely pure;

(4) the functor $\operatorname{Mod} \mathcal{P} \longrightarrow \operatorname{Mod} \mathcal{R}=(\operatorname{Mod} R)^{\mathbf{Z}}=\prod_{\mathbf{z}} \operatorname{Mod} R$ sending an object $F$ of $\operatorname{Mod} \mathcal{P}$ to $(F(R[n]))_{n \in \mathbf{Z}}$ is an equivalence of categories.

If the equivalent conditions (1)-(4) hold, then every complex $X$ in $D(R)$ is homologically isomorphic to the coproduct of the cohomology groups $\coprod_{n \in \mathbf{Z}} H^{n}(X)[-n]$. Each of these is an absolutely pure $R$-module.

Proof. (1) $\Longrightarrow(2)$. From $[\mathrm{GaPr}]$ it follows that a complex $X$ is injective in $D(R)$ iff all its cohomology groups $H^{n}(X)$ are injective modules. Then use Proposition 7.1 and the fact that a ring is von Neumann regular iff every (indecomposable) pure-injective module is injective.

$(2) \Longrightarrow(3)$. A complex $X$ is absolutely pure iff there is an injective object $Q$ and a pure monomorphism $X \longrightarrow Q$. By assumption, a pure-injective hull of an arbitrary complex $X$ is injective. 
$(3) \Longrightarrow(1)$. Let $M$ be an $R$-module; then the complex $M[0]$ is absolutely pure. By Theorem 5.2 $M=H^{0}(M[0])$ is an absolutely pure module and therefore $R$ is von Neumann regular.

$(2) \Longleftrightarrow(4)$. The category $\operatorname{Mod} \mathcal{R}$ is the quotient category of $\operatorname{Mod} \mathcal{P}$ with respect to the localizing subcategory $\mathcal{S}=\{F \in \operatorname{Mod} \mathcal{P} \mid F(R[n])=0$ for all $n \in \mathbf{Z}\}$. The composed functor $\operatorname{Mod} \mathcal{P} \stackrel{(-) s}{\longrightarrow} \operatorname{Mod} \mathcal{P} / \mathcal{S} \stackrel{\sim}{\longrightarrow} \operatorname{Mod} \mathcal{R}$ is an equivalence iff every injective object in $\operatorname{Mod} \mathcal{P}$ is $\mathcal{S}$-closed. The injectives of $\operatorname{Mod} \mathcal{P}$ are of the form $H_{X}$ with $X$ a pure injective complex and the $\mathcal{S}$-closed injectives are the objects of the form $H_{Q}$ with $Q$ an injective complex, so the equivalence is clear.

The fact that every complex $X$ in $D(R)$ is homologically isomorphic to the coproduct of the cohomology groups $\coprod_{n \in \mathbf{Z}} H^{n}(X)[-n]$ and that each of them is an absolutely pure $R$-module follows from Theorem 5.2 (every von Neumann regular ring is coherent).

Corollary 8.3. Let $R$ be a von Neumann regular ring. A complex in $D(R)$ is pure-injective iff it is a complex of the form $\bigsqcup_{n \in \mathbf{Z}} Q^{n}[-n]$ with the $Q^{n}$ injective modules.

Proof. By $[\mathrm{GaPr}]$ injective complexes are the complexes of the form $\coprod_{n \in \mathbf{Z}} Q^{n}[-n]$ with $Q^{n}$ injective modules. The preceding theorem implies the claim.

By $[\mathrm{He} 2,4.4]$ a ring $R$ is von Neumann regular iff the functor $M \longmapsto M \otimes_{R}-$ from $\operatorname{Mod} R$ to $\mathcal{C}_{R}$ is an equivalence. Let $J$ be a two-sided ideal of a von Neumann regular ring $R$ and denote by $\mathcal{S}_{J}$ the Serre subcategory of $\bmod R \simeq \mathrm{fp}_{R}$ which consists of finitely generated summands of coproducts of finitely many copies of $J$, that is,

$$
\mathcal{S}_{J}=\left\{P \in \bmod R|P| J^{(n)} \text { for some } n\right\} .
$$

Then $\mathcal{S}_{J}=\mathcal{S}(R / J)=\left\{P \in \bmod R \mid \operatorname{Hom}_{R}(P, R / J)=0\right\}$ [He2, 4.5]. Moreover, the maps

$$
\mathcal{S} \longmapsto t_{\mathcal{S}}(R) \quad \text { and } \quad J \longmapsto \mathcal{S}(R / J)
$$

give an inclusion-preserving bijective correspondence between the Serre subcategories $\mathcal{S}$ of $\bmod R \simeq \mathrm{fp}_{R}$ and the two-sided ideals $J$ of $R$ [He2, 4.6].

Thus the open subsets of the Ziegler spectrum of a von Neumann regular ring have the form

$$
\mathcal{O}(J)=\left\{E \in \operatorname{Zsp} R \mid \operatorname{Hom}_{R}(J, E) \neq 0\right\},
$$

where $J$ is a two-sided ideal.

Consider now the category $\operatorname{Mod} \mathcal{R}=(\operatorname{Mod} R)^{\mathbf{Z}}$. Every Serre subcategory $\mathcal{S}$ of $\bmod \mathcal{R}$ may be viewed as a $\mathbf{Z}$-tuple $\left(\mathcal{S}_{n}\right)_{n \in \mathbf{Z}}$ of Serre subcategories of $\bmod R$, one in each degree.

Theorem 8.4 (cf. [He2]). Let $R$ be a von Neumann regular ring. There is an inclusionpreserving bijective correspondence between the Serre subcategories $\mathcal{S}=\left(\mathcal{S}_{n}\right)_{n \in \mathbf{Z}}$ of $\bmod \mathcal{P} \simeq$ $\bmod \mathcal{R}$ and the $\mathbf{Z}$-tuples $\left(J_{n}\right)_{n \in \mathbf{Z}}$ of two-sided ideals $J_{n}$ of $R$ given by the maps

$$
\left(\mathcal{S}_{n}\right)_{n \in \mathbf{Z}} \longmapsto\left(t_{\mathcal{S}_{n}}(R)\right)_{n \in \mathbf{Z}} \quad \text { and } \quad\left(J_{n}\right)_{n \in \mathbf{Z}} \longmapsto\left(\mathcal{S}\left(R / J_{n}\right)\right)_{n \in \mathbf{Z}}
$$

which are mutually inverse.

We are now in a position to prove the following theorem. 
Theorem 8.5. Let $R$ be a von Neumann regular ring. The Ziegler spectrum of the derived category $D(R)$ is homeomorphic to the disjoint union

$$
\operatorname{Zsp} D(R) \simeq \bigsqcup_{n \in \mathbf{Z}} \mathrm{Zsp} R
$$

of countably many copies of Zsp R. Moreover, the open subsets of the Ziegler spectrum have the form

$$
\mathcal{O}\left(\left(J_{n}\right)_{n \in \mathbf{Z}}\right)=\left\{E \in \mathbf{Z s p} D(R) \mid D(R)\left(J_{n}[-n], E\right) \neq 0 \text { for some } n \in \mathbf{Z}\right\}
$$

where $\left(J_{n}\right)_{n \in \mathbf{Z}}$ is a $\mathbf{Z}$-tuple of two-sided ideals of $R$.

Proof. By Corollary 8.3 every pure-injective complex is of the form $\coprod_{n \in \mathbf{Z}} Q^{n}[-n]$ with the $Q^{n}$ injective modules. Since any von Neumann regular ring is coherent and any finitely presented module is projective, the proof of Theorem 8.1 applies to show that the Ziegler spectrum of the derived category $D(R)$ is homeomorphic to the disjoint union

$$
\text { Zsp } D(R) \simeq \bigsqcup_{n \in \mathbf{Z}} \text { Zsp } R
$$

of countably many copies of Zsp $R$.

The open subsets of the Ziegler spectrum Zsp $R$ of $R$ are of the form $\mathcal{O}(J)$ with $J$ a two-sided ideal of $R$ (see above). It follows that the open subsets of $\operatorname{Zsp} D(R)$ are of the form (4).

\section{References}

[Au M. Auslander, Coherent functors, Proc. Conf. on Categorical Algebra (La Jolla, CA, 1965), Springer, New-York, 1966, 189-231.

[Bel1] A. Beligiannis, Relative homological algebra and purity in triangulated categories, J. Algebra 227(1) (2000), 268-361.

[Bel2] $147-185$

[BK] D. Benson, H. Krause, Pure injectives and the spectrum of the cohomology ring of a finite group, J. reine angew. Math. 542(1) (2002), 23-51.

[CKN] J. D. Christensen, B. Keller, A. Neeman, Failure of Brown representability in derived categories, Topology 40(6) (2001), 1339-1361.

[ES] P. Eklof, G. Sabbagh, Model-completions and modules, Ann. Math. Logic 2 (1971), 251-295.

[G] G. Garkusha, Grothendieck categories, Algebra i Analiz 13(2) (2001), 1-68. (Russian). English transl. in St. Petersburg Math. J. 13(2) (2002), 149-200.

[GaPr] G. Garkusha, M. Prest, Injective objects in triangulated categories, preprint, 2002.

[He1] I. Herzog, Elementary duality of modules, Trans. Amer. Math. Soc. 340(1) (1993), 37-69.

[He2] The Ziegler spectrum of a locally coherent Grothendieck category, Proc. London Math. Soc. 74(3) (1997), 503-558.

[HPS] M. Hovey, J. H. Palmieri, N. P. Strickland, Axiomatic stable homotopy theory, Mem. Amer. Math. Soc. 128 (1997), No. 610.

[JeLe] C. U. Jensen, H. Lenzing, Model theoretic algebra: with particular emphasis on fields, rings, modules, Algebra, Logic and Appl. 2, New York etc., Gordon and Breach, 1989.

[Kr1] H. Krause, The spectrum of a locally coherent category, J. Pure Appl. Algebra 114(3) (1997), 259-271.

[Kr2] Decomposing thick subcategories of the stable module category, Math. Ann. 313(1) (1999), 95-108.

[Kr3] Smashing subcategories and the telescope conjecture - an algebraic approach, Invent. Math. 139(1) (2000), 99-133.

[Kr4] Coherent functors in stable homotopy theory, Fundamenta Math. 173(1) (2002), 33-56.

[KR] H. Krause, U. Reichenbach, Endofiniteness in stable homotopy theory, Trans. Amer. Math. Soc. 353(1) (2001), 157-173.

[Ne1] A. NeEman, The Brown representability theorem and phantomless triangulated categories, J. Algebra 151(1) (1992), 118-155. 
[Ne2] The Grothendieck duality theorem via Bousfield's techniques and Brown representability, J. Amer. Math. Soc. 9(1) (1996), 205-236.

[PrPu] M. Prest, G. Puninski, Some model theory over hereditary noetherian domains, J. Algebra 211(1) (1999), 268-297.

[St] B. Stenström, Rings of quotients, Grundlehren math. Wiss. 217, Berlin-Heidelberg-New York, Springer-Verlag, 1975.

[Zie] M. Ziegler, Model theory and modules, Ann. Pure Applied Logic 26 (1984), 149-213.

St. Petersburg State University, Department of Mathematics and Mechanics, Universitetsky PR. 28, 198504, Stary Peterhof, Russia

E-mail address: ggarkusha@mail.ru

Department of Mathematics, University of Manchester, Oxford Road, Manchester, M13 9PL, ENGLAND

E-mail address: mprest@maths.man.ac.uk 